\title{
Mapping Childcare Support for Patients at North American Hospitals and Cancer Centres: An Environmental Scan.
}

Katherine Preston ( $\nabla$ katepres@student.ubc.ca )

The University of British Columbia Faculty of Medicine https://orcid.org/0000-0003-3413-6697

Mackenzie MacDonald

BC Cancer Agency Vancouver Centre

Meredith Giuliani

Princess Margaret Hospital: Princess Margaret Hospital Cancer Centre

Bonnie Leung

BC Cancer Agency Vancouver Centre

Christine Simmons

BC Cancer Agency Vancouver Centre

Barbara Melosky

BC Cancer Agency Vancouver Centre

Paris-Ann Ingledew

BC Cancer Agency Vancouver Centre

\section{Research Article}

Keywords: on-site childcare, hospital childminding, supervised playrooms, parents with cancer, psychosocial support

Posted Date: June 7th, 2021

DOl: https://doi.org/10.21203/rs.3.rs-556117/v1

License: (c) (i) This work is licensed under a Creative Commons Attribution 4.0 International License. Read Full License

Version of Record: A version of this preprint was published at Supportive Care in Cancer on August 4th, 2021. See the published version at https://doi.org/10.1007/s00520-021-06460-x. 


\section{Abstract \\ Purpose}

Approximately one quarter of cancer patients are parents to young children. One unique challenge faced by this patient group is the difficulty of accessing childcare support during medical appointments. Hospital-based childcare options could represent a solution to this problem, but to this point, no comprehensive scans have described existing on-site childcare centres. The purpose of this study is to identify and characterize on-site childcare services available to patients at North American hospitals. This information could inform the development of similar programs for cancer patients.

\section{Methods}

Using publicly available information, an environmental scan of the grey literature was conducted to investigate Canadian and American hospitals for the presence of childcare services. A standardized data collection tool was used to extract centre characteristics.

\section{Results}

Twenty-six childcare centres were identified across 161 hospitals. The majority of the centres were associated with pediatric hospitals (77\%) and were located in the United States (69\%). Only a single childcare centre was associated with a cancer hospital. All centres accommodated children between the ages of 3 and 8 . Most centres were open for over 30 hours per week (77\%) and were free of charge to users (89\%). Other characteristics, including capacity and staffing, varied widely.

\section{Conclusions}

These results represent an inventory of patient-accessible, on-site childcare services currently available at North American hospitals. Cancer patients who are also parents may especially need this kind of support, as they are at high risk for financial toxicity.

\section{Introduction}

The National Cancer Institute estimates $20 \%$ of newly diagnosed cancer patients are between the typical parenting ages of 20 and 54 [1]. Not only are parental cancer rates appreciable, but they have the potential to rise; the average age of childbearing women has increased in recent years, and older parents are at a greater risk of cancer as compared to their younger counterparts [1-3].

Cancer patients who are also parents face significant psychosocial stress. These patients face an internal struggle in balancing their own needs with those of their children, and the common response to prioritize 
the latter leads to exhaustion and burnout [4-6]. Many patients feel pressured to maintain a sense of routine and normalcy for their children despite changing circumstances, and mothers, especially, tend to feel obligated to continue to take responsibility for childcare and housekeeping, despite their illness [9]. As well, it is common for parents to feel guilt due to their perception that their lower energy and physical limitations - often caused by both their illness and treatment - prevent them from being 'good parents' [5,7-10]. There are additional existential worries for parents with cancer, such as the fear that they may not live long enough to raise their children to adulthood [11]. Even when in remission, patients who have children fear recurrence more than patients who do not [12].

While the psychosocial stress burden on parents with cancer cannot be easily alleviated, examining solutions to practical challenges such as lack of childcare may have benefit for patients, their families, and the system. Many studies have suggested that the difficulty associated with coordinating childcare and health care appointments contributes to role strain, frustration, and appointment noncompliance for patients $[7-8,10,13]$. To our knowledge, only one study has specifically assessed childcare needs in cancer patients and the success of a supportive childcare intervention [13]. This study, conducted by Cohen et al., found that over $50 \%$ of mothers considered childcare to be their most overwhelming responsibility after their cancer diagnosis, and $75 \%$ indicated that a supportive childcare intervention allowed them to keep appointments that they would have otherwise had to miss [13]. On-site childcare also has the potential benefit of convenience for patients and the ability to link families in with other oncology services.

While the evidence for the need for childcare support is compelling, existing literature on supportive cancer care has not been overly focused on this need, nor the degree to which it has actually been met. The aim of this study is to identify and describe on-site childcare services available to patients at hospitals across Canada and the United States (U.S.). This inventory is intentionally limited to layaccessible searching avenues, to allow for a description of childcare options that could reasonably be identified by patients prior to hospital visits, based on publicly available information. The results of this study provide a description of the landscape of currently available services to patients at North American hospitals and may allow this knowledge to translate into services at cancer centers.

\section{Methods}

An environmental scan based on a search of the grey literature was conducted to assess the current availability of on-site childcare services for patients at hospitals across Canada and the U.S. This method was selected to simulate information that would be accessible to patients looking for on-site childcare options at hospitals. This scan involved two stages: (1) the selection of hospitals for inclusion in the study and (2) a description of the details of existing childcare options at these hospitals.

\section{Hospital Selection}


The first step in hospital selection was the identification of broad hospital types to investigate: (1) university hospitals, (2) cancer centres, (3) pediatric hospitals, and (4) women's hospitals. A shortlist of hospitals for each type from both the U.S. and Canada was compiled to represent a broad distribution of

hospitals that were geographically spread, and either associated with academic activities or deemed to be "highly ranked". Noting that this would not be an exhaustive list of all hospitals, this selection process was based on the assumption that comprehensive hospitals and highly rated hospitals - especially when patient satisfaction was considered in ratings - would be more likely to provide services impacting patient experience, such as childcare. The shortlist was composed by reviewing published lists of hospitals, as outlined below.

For the Canadian university hospitals, one large (defined as having a level 1 trauma centre) teaching hospital associated with each Canadian medical school was chosen, which allowed for the identification of 16 university hospitals. A comprehensive list of Canadian cancer centres from the Canadian Organization of Medical Physicists was utilized to identify 28 Canadian cancer centres. Only 11 dedicated pediatric hospitals and 5 women's hospitals were identified in Canada, all of which were included. In total, 60 Canadian hospitals were included in the original shortlist.

For the American university hospitals, the top 30 hospitals associated with medical schools from Newsweek's "Top 100 Hospitals in the USA" list were selected, with 30 representing a manageable number of highly ranked hospitals [15]. For the cancer hospitals, the U.S. News \& World Report's top-rated NCldesignated comprehensive cancer centres in each state were chosen, leaving 29 cancer hospitals in total $[16,17]$. For pediatric hospitals, the top 30 hospitals across different pediatric specialties were selected, according to the U.S. News \& World Report [18]. For the American women's hospitals, the 15 most highly ranked gynecology hospitals, which had not already been accounted for, were chosen [19]. In total, 104 American hospitals were included in the original shortlist.

The original shortlist was then modified in an iterative process. There were some instances where two different types of hospitals were located on the same physical site. One of two such hospitals was eliminated from the list as a duplicate if (1) the two hospitals were located at the same address and (2) separate amenities were not listed online for the two hospitals. Additionally, some hospitals were added to the list as they were identified during the investigative process, typically through relationships to hospitals on the original shortlist. After these modifications were made, the final shortlist included 55 Canadian hospitals (13 university hospitals, 28 cancer hospitals, 10 pediatric hospitals, 3 women's hospitals, and 1 community hospital), and 106 American hospitals (27 university hospitals, 29 cancer hospitals, 33 pediatric hospitals, 15 gynecology hospitals, and 2 community hospitals), with community hospitals being defined as small, non-federal, general hospitals [20] (Supplementary Material 1).

\section{Identification of On-Site Childcare Centres and Data Extraction}

For each hospital included in this analysis, a systematic approach, as outlined below, was used to firstly determine if on-site childcare options were available, and to secondly extract key characteristics of 
identified centres.

Firstly, each hospital's official website was hand-searched for mention of relevant resources (e.g. childcare centres, patient amenities, playrooms, etc.). This involved both searching the website's database and using the website's search bar by entering keywords (childcare, child life, playroom, daycare, sibling care). All website searches were conducted in the month of May 2020. In some cases, relevant documents (e.g. web pages, reports) describing on-site childcare services were identified. The URL of these individual records were recorded, and if available, the following data was extracted from these sources using an Excel spreadsheet: the centre name, hospital affiliation, location, hours of operation, users, capacity, age range, limits on use, staff, registration requirements and drop-in potential, cost for users, and funding structure. In some cases, not all of this data was available from the website alone, and so a second Google search was performed to identify alternate sources of information about the resource, such as newspaper articles. When a contact phone number for a centre or service was identified, a recorded phone conversation was made. Where possible, these alternate sources of information allowed for the extraction of the remaining data characteristics.

When hospital websites explicitly expressed that on-site childcare was not available, this information was recorded. However, if the absence of a centre could not be confirmed from a hospital's website alone, the next step was to call the hospital directly, to confirm that no on-site childcare options had been missed. The first phone number that was called was always the telephone operator, and, if needed, phone calls to departments such as child life, social work, or patient services were subsequently made. Where centres were present, these phone conversations allowed for information about the centres to be collected, which was recorded on the Excel spreadsheet. In some cases, these phone conversations led to email correspondences, which allowed for the extraction and recording of data in an analogous manner.

The grey literature search was conducted with the aim of recording as many on-site childcare options as possible. The next step was to narrow down those options, using inclusion criteria, in order to identify accessible locations where patients could realistically drop off their children for short term supervision while attending medical appointments. To be included, the childcare centres had to correspond to the following criteria: (a) be hospital affiliated, (b) be consistently supervised, (c) be a physical centre, either

on-site or nearby, (d) be realistically accessible to patients with children or siblings of patients being one of the main user groups, and (e) be available for a short term period (i.e. not a formal daycare centre requiring yearlong registration).

\section{Results}

\section{Environmental Scan}

The flow scheme of the environmental scan is presented in Fig. 1. In May 2020, 26 patient-accessible, onsite childcare centres were identified at hospitals across Canada and the U.S., based on inclusion criteria.

\section{Characteristics of Centres Found}


The main characteristics of the centres retained based on the inclusion criteria are summarized in Table 1, and full details are provided in Supplementary Material 2.

\section{i. Hospital Characteristics}

While childcare centres were identified at all types of hospitals explored, the overwhelming majority of centres $(77 \%)$ were associated with pediatric hospitals. Centres were identified in both Canada and the U.S., with 8/55 Canadian hospitals and 18/106 American hospitals having patient-accessible on-site services.

\section{ii. General Operational Characteristics}

Hours of operation varied across centres: approximately half of the centres were open between 30 to 45 hours per week (46\%), approximately one third of the centres were open between 46 and 60 hours per week (31\%), and $15 \%$ of centres were open for fewer than 30 hours per week. The remaining centres (8\%) had variable hours, which depended on factors such as staff availability. Centres open for less than 45 hours per week tended to have hours of operation restricted to weekdays, while centres open for more than 45 hours per week were more likely to have hours of operation on all days of the week (Fig. 2). Generally, all centres' hours of operation tended to fall between 8:00 am and 6:00 pm (Supplementary Material 2).

In terms of user groups, most centres catered exclusively to children associated with patient families. Specifically, $46 \%$ of centres were intended exclusively for the use of siblings of pediatric patients, children of adult patients, or both, while $39 \%$ of centres were intended for use by these groups as well as pediatric patients. The remaining centres served a broader group of children, including those associated with visitor or employee families.

In terms of staffing, over half of the centres were supervised by a combination of volunteers and staff members (58\%). Many centres were supervised exclusively by staff members (39\%), while a single centre was found to be supervised exclusively by trained volunteers.

Funding structure also varied across centres, with 35\% of centres being funded by a hospital or child life foundation, $39 \%$ by charitable foundations or private donors, and $12 \%$ by some combination of these sources of income. Two centres that charged users for their services used this revenue to support the centre, along with supplementation from hospital operational funds (8\%).

\section{iii. Operational Characteristics Affecting Accessibility}

The capacity of centres varied widely, with $42 \%$ of centres accommodating 11 to 30 children, $19 \%$ accommodating 10 children or fewer, and 8\% accommodating between 31 and 40 children at maximum capacity. Some centres had variable capacities that depended on staff and volunteer availability (15\%).

Age ranges of children served varied widely, with some centres only specifying a lower age limit, ranging from 15 months to 3 years old (12\%), and others only specifying an upper age limit, ranging from 10 to 13 
years old (27\%). Forty-six percent of centres specified both an upper and lower limit age range, with the majority of these centres accommodating children between the ages of 3 and 8 years (Fig. 3). Three centres $(12 \%)$ were accessible to all ages.

Many centres specified time-based limits on usage, with about a third of centres having a limit between 1.5 to 2 hours of usage per day (35\%). Fifteen percent of centres had a limit of 3 to 4 hours of usage per day, while $35 \%$ of centres had no formal limitation on usage. Two centres specified a limit of days per fiscal year that the centre could be used, rather than an hourly limit, and one centre indicated that limitation on use was staff dependent. Registration varied across centres, with the majority of the centres operating on a drop-in, first-come, first-served model (54\%). Thirty-one percent of centres encouraged preregistration of children with drop-in services as available, and $12 \%$ of centres operated exclusively on a pre-registration basis.

The vast majority of centres identified did not charge for use (89\%), but three centres charged a nominal, time-based fee (12\%). 
Table 1

Characteristics of on-site childcare programs.

Scan $(\mathrm{N}=26) \mathrm{n}$ $(\%)^{a}$

\section{Characteristics}

\section{Affiliated Hospital Type}

University Hospital

Cancer Centre

Womens' Hospital

Pediatric Hospital

Community Hospital/Health Authority

Womens' and Cancer Hospital

\section{Location}

Canada

United States

\section{Hours of Operation}

$<30$ hours total

$30-45$ hours total

46-60 hours total

Variable Hours
$1(4)$

$1(4)$

$1(4)$

$20(77)$

2 (8)

$1(4)$

$8(31)$

$18(69)$

$4(15)$

$12(46)$

$8(31)$

$2(8)$

\section{Users}

Children or siblings of patients only (no patients)

Children or siblings of patients and pediatric patients

Children or siblings of patients, pediatric patients, children of visitors, and children of employees

\section{Capacity}

10 children or fewer

Between 11 and 30 children

Between 31 and 40 children

${ }^{\text {a}}$ Totals of each category may not equal $100 \%$ due to rounding.

bThis information was not retrievable for all centres using the methods described above (handsearching of websites, targeted phone calls, and email correspondences) 


\section{Characteristics}

Scan $(\mathrm{N}=26) \mathrm{n}$

$(\%)^{a}$

Variable; volunteer/staff dependent

4 (15)

Unspecified*

$4(15)$

\section{Age Range}

Only lower limit age specified (ranging from 15 months -3 years)

$3(12)$

Only upper limit age specified (ranging from 10-13 years)

$7(27)$

Lower limit age specified (ranging from 6 weeks -3 years) and upper limit age specified (ranging from 8-13 years)

Accessible to all ages

Unspecified**

$3(12)$

\section{Limits on Use}

1.5-2 hours maximum per day

3-4 hours maximum per day

20 days maximum per fiscal year, no hourly limit

No limits on use

Volunteer-dependent limits

Unspecified**

\section{Supervising Staff}

Trained volunteers only

Trained volunteers and staff (child life specialists, childhood educators, etc.)

Trained staff only

\section{Registration Options}

Pre-registration only

Pre-registration, with drop-in as available

Drop-in only

Unspecified**

aTotals of each category may not equal $100 \%$ due to rounding.

bThis information was not retrievable for all centres using the methods described above (handsearching of websites, targeted phone calls, and email correspondences) 


\section{Cost for Users}

Free

Time-based charges

\section{Funding Structure}

Hospital or Child Life Foundation

Charitable foundation and/or private donors (exclusively philanthropic funding)

Combined funding: Hospital foundation/operational funds and private donations

Revenue-based with supplementation from hospital operational funds

Unspecified $^{b}$

${ }^{\text {a }}$ Totals of each category may not equal $100 \%$ due to rounding.

bThis information was not retrievable for all centres using the methods described above (handsearching of websites, targeted phone calls, and email correspondences)

\section{Discussion}

As the median age of parenting increases, so does the proportion of cancer patients who are also parents. The undeniable psychosocial stress experienced by these patients has the potential to be mitigated by instrumental supports, such as on-site childcare services at cancer hospitals. As such, this environmental scan of the grey literature aimed to characterize the current state of on-site childcare options for patients at North American hospitals. Based on inclusion criteria, 26 patient-accessible, on-site childcare centres were identified in an investigation of 161 hospitals. Three notable trends emerged from the characteristics of these centres.

The majority of centres (69\%) were found in the U.S. More American hospitals than Canadian hospitals were explored (106 vs. 55), but a greater proportion of American hospitals had on-site childcare centres compared to their Canadian counterparts, even after accounting for this. One possible explanation for this finding is that the health care funding structures in the U.S. and Canada differ significantly, and generally, national health care spending is more significant in countries with more privatized systems, such as the U.S. [21]. This spending could translate to funding for on-site childcare, with one important use for funding being the staffing of childcare centres. Approximately half (10 of 19) of the American centres rely entirely on paid staff, while all but one of the Canadian centres rely partially or entirely on volunteer workers (Table 1; Supplementary Material 2). This observation highlights that while a privatized system may create funding that facilitates the creation of on-site childcare centres, it is not an absolute necessity, 
especially when other valuable resources (such as volunteers) can be utilized. Therefore, on-site hospital childcare can be a reality in both private-leaning and public-leaning systems.

Another trend that emerged was that most of the identified centres are located in pediatric hospitals, and so are intended for siblings of patients. Most of these centres are associated with child life departments. Child life services at pediatric hospitals help to prioritize a family-centered approach to patient care, which involves the heavy consideration of familial and sibling needs [22, 23]. Some of these needs can be met by on-site childcare centres; for example, the practical need for child supervision, and the emotional need for respite for both parents and siblings [22,23]. Adult patients who are parents are also in need of instrumental support, particularly outpatients with frequent and variable appointment schedules, such as cancer patients. Unfortunately, the lack of on-site childcare services at adult hospitals indicates that, in some ways, the needs of this demographic have potentially been overlooked. Therefore, the fact that $77 \%$ of identified centres were found in pediatric hospitals can be generalized to suggest that when family needs are prioritized, on-site childcare services are more likely to be offered.

In addition to highlighting trends towards American and pediatric hospital-associated childcare centres, the majority (89\%) of explored centres are free of charge to users. Charging money for an on-site service has its advantages: two of the centres that charged a user fee reported that this revenue was used entirely to fund the centres' operations, and, correspondingly, these centres had some of the most extensive hours of operation and largest capacities of all centres explored. Therefore, a small fee could make the existence of on-site hospital childcare centres more financially feasible, and could allow existing centres to offer more extensive services.

However, charging for on-site childcare can create a barrier to access for patients, especially patients with chronic illnesses, such as cancer, who require multiple appointments and might therefore rely heavily on such services. In recent years, costs associated with cancer have significantly increased both directly (i.e. treatment costs) and indirectly (i.e. lost productivity at work), causing cancer to rank as the second most expensive disease in the U.S. [24]. This has the potential to create financial toxicity, leading to substantial financial burden and thus reduced quality of life, increased distress, and worse patient outcomes [24]. Among cancer patients, one group that has been identified as being disproportionately affected by financial toxicity are younger patients (20-65 years old), as these patients tend to have lower savings and fewer assets [24]. This age group is also the most likely to comprise of parents. As such, while charging for the use of an on-site childcare service can be advantageous, financial toxicity to patients should not be overlooked as a potential adverse effect, especially in cancer hospitals.

The present findings are limited by several factors. Firstly, it is possible that some on-site hospital childcare options in North America were missed in our scan, as only 161 hospitals were ultimately explored. The rationale for limiting the environmental scan to this shortlist was based on the knowledge that, to date, no other scan has evaluated on-site childcare hospitals in North America. As the first of its kind, this scan could not reasonably assess every hospital in Canada and the U.S. In order to make the scan a manageable undertaking, top-ranked hospitals were explored, based on the assumption that on-

Page $11 / 17$ 
site childcare centres were more likely to be found at highly ranked hospitals. However, this assumption may not be true, and therefore, it may be worth expanding the list of hospitals in future studies. Further, six hospitals were added to the list as they were identified during the investigative process. It is possible that the addition of these hospitals weakens the generalizability of the scan's results, but it is worth noting that these hospitals had a connection to the original list and did not represent a majority of hospitals on the final shortlist. It is also possible that some on-site childcare options were missed, even within the list of hospitals explored, such as more informal childminding services not advertised on hospital websites. Similarly, there were childcare centres which were identified, but could not be completely characterized according to the descriptors in Table 1, based on publicly available information. However, the lay-accessible searching methods used to conduct this scan ensured that those services identified could reasonably be identified by patients prior to hospital visits, based on publicly available information. Therefore, if some on-site childcare services, or their characteristics, were missed by our scan, this suggests that this information is perhaps not adequately accessible to patients.

Finally, these findings have implications for future research in this area. While this inventory is a starting point, it did not determine the degree to which on-site childcare supports for cancer patients are actually needed. Future research should evaluate the perspectives of current cancer patients on this topic, as this knowledge would help to expand current understanding of the instrumental support needs of this patient group, and could inform the development of future childcare centres.

\section{Conclusion}

Cancer patients who are also parents may especially benefit from on-site childcare at hospitals, as this support could reduce the financial toxicity and distress that disproportionately affects this patient population. In evaluating the state of existing supports, this environmental scan identified and characterized 26 patient-accessible, on-site childcare services at hospitals across North America using lay-accessible searching methods. The scan indicated that the majority of hospitals explored had no such services, that existing services were mostly represented in American, pediatric hospitals, and that childcare supports were underrepresented in cancer hospitals. Future studies should focus on better characterizing the need for in-hospital childcare supports in the cancer patient population.

\section{Declarations}

Funding: The authors did not receive support from any organization for the submitted work.

Conflicts of Interest: BL has previously received a speaker honorarium from Takeda. MG is a member of advisory boards for AstraZeneca and Bristol Myers.

Availability of data and material: All data generated or analyzed during this study are included in this article and its supplementary material files. 
Code availability: The software diagrams.net was used to create Figure 1. Figures 2 and 3 were created using Microsoft Excel.

Author contributions: All authors contributed to the study conception and design. Shortlist creation and environmental scan were performed by Katherine Preston and Mackenzie MacDonald. The first draft of the manuscript was written by Katherine Preston, and all authors commented on previous versions of the manuscript. All authors read and approved the final manuscript.

Ethics approval: Not applicable.

Consent to participate: Not applicable.

Consent for publication: Not applicable.

\section{References}

1. Howlader N, Noone AM, Krapcho M, Miller D, Brest A, Yu M, Ruhl J, Tatalovich Z, Mariotto A, Lewis DR, Chen HS, Feuer EJ, Cronin KA (2020) SEER Cancer Statistics Review, 1975-2017. National Cancer Institute. https://seer.cancer.gov/csr/1975_2017/. Accessed June 302020.

2. Statistics Canada (2017) Fertility: Fewer Children, Older Moms. https://www150.statcan.gc.ca/n1/pub/11-630-x/11-630-x2014002-eng.htm. Accessed June 30 2020.

3. Mathews TJ, Hamilton BE (2016) Mean Age of Mothers is on the Rise: United States, 2000-2014. NCHS Data Brief. 232:1-8. https://www.cdc.gov/nchs/products/databriefs/db232.htm

4. Golby BJ (2014) Parenting with chronic cancer: a relational perspective. Social work in health care 53(1):48-58. https://doi.org/10.1080/00981389.2013.832468

5. Elmberger E, Bolund C, Magnusson A, Lützén K, Andershed B (2008) Being a mother with cancer: achieving a sense of balance in the transition process. Cancer nursing, 31(1):58-66. https://doi.org/10.1097/01.ncc.0000305677.90963.67

6. Helseth S, Ulfsæt N (2005) Parenting experiences during cancer. Journal of advanced nursing 52(1):38-46. https://doi.org/10.1111/j.1365-2648.2005.03562.x

7. Semple CJ, McCance T (2010) Parents' experience of cancer who have young children: a literature review. Cancer nursing: 33(2):110-118. https://doi.org/10.1097/NCC.0b013e3181c024bb

8. Fisher C, O'Connor M (2012) "Motherhood" in the context of living with breast cancer. Cancer Nursing 35(2):157-163. https://doi.org/10.1097/NCC.0b013e31821cadde

9. Ohlén J, Holm AK (2006) Transforming desolation into consolation: being a mother with lifethreatening breast cancer. Health care for women international 27(1):18-44. https://doi.org/10.1080/07399330500377226

10. Rashi C, Wittman T, Tsimicalis A, Loiselle CG (2015) Balancing illness and parental demands: coping with cancer while raising minor children. Oncol Nurs Forum 42(4):337-344. 
https://doi.org/10.1188/15.0NF.337-344

11. Dunn J, Steginga SK (2000) Young women's experience of breast cancer: defining young and identifying concerns. Psycho-Oncology 9(2):137-146. https://doi.org/10.1002/(sici)10991611(200003/04)9:2<137::aid-pon442>3.0.co;2-0

12. Arès I, Lebel S, Bielajew C (2014) The impact of motherhood on perceived stress, illness intrusiveness and fear of cancer recurrence in young breast cancer survivors over time. Psychol Health 29(6):651670. https://doi.org/10.1080/08870446.2014.881998

13. Cohen L, Schwartz N, Guth A, Kiss A, Warner E (2017) User survey of Nanny Angel Network, a free childcare service for mothers with cancer. Curr Oncol 24(4):220. https://doi.org/10.3747/co.24.3512

14. Canadian Organization of Medical Physicists (2020) Canadian Cancer Centres. https://www.compocpm.ca/english/career-education/career-resources/canadian-cancer-centres.html Accessed April 26 2020.

15. Bates DW, Kaplan GS, Zimlichman E, Meier CA, Katz G, Deerberg-Wittram J (2020) Best Hospitals USA. Newsweek. https://www.newsweek.com/best-hospitals-2020/united-states Accessed April 26 2020.

16. S. News Staff (2020) Best Hospitals for Cancer 2019-2020. U.S. News and World Report. https://health.usnews.com/best-hospitals/rankings/cancer Accessed April 272020.

17. National Cancer Institute (2020) Find an NCl-Designated Cancer Center. https://www.cancer.gov/research/infrastructure/cancer-centers/find Accessed April 272020.

18. S. News Staff (2020) Best Children's Hospitals 2019-2020. US News \& World Report. https://health.usnews.com/best-hospitals/pediatric-rankings Accessed April 272020.

19. S. News Staff (2020) Best Hospitals for Gynecology 2019-2020. US News \& World Report. https://health.usnews.com/best-hospitals/rankings/gynecology Accessed April 272020.

20. American Hospital Association (2021) Fast Facts on US Hospitals, 2021. https://www.aha.org/statistics/fast-facts-us-hospitals Accessed January 152021.

21. Gohmann SF (2010) A comparison of health care in Canada and the United States: the case of Pap smears. Med Care 48(11):1036-40. https://doi.org/10.1097/MLR.0b013e3181eb31d2.

22. Ludmir J, Netzer G (2019) Family-Centered Care in the Intensive Care Unit-What Does Best Practice Tell Us? Semin Respir Crit Care Med 40(5):648-654. https://doi.org/10.1055/s-0039-1697957

23. Brown C, Chitkara MB (2014) Policy Statement: Child Life Services. Pediatrics 133(5):1471-78. https://doi.org/10.1542/peds.2014-0556

24. Snyder RA, Chang GJ (2019) Financial toxicity: a growing burden for cancer patients. Bulletin of the American College of Surgeons. https://bulletin.facs.org/2019/09/financial-toxicity-a-growing-burdenfor-cancer-patients/. Accessed January 152021.

\section{Figures}




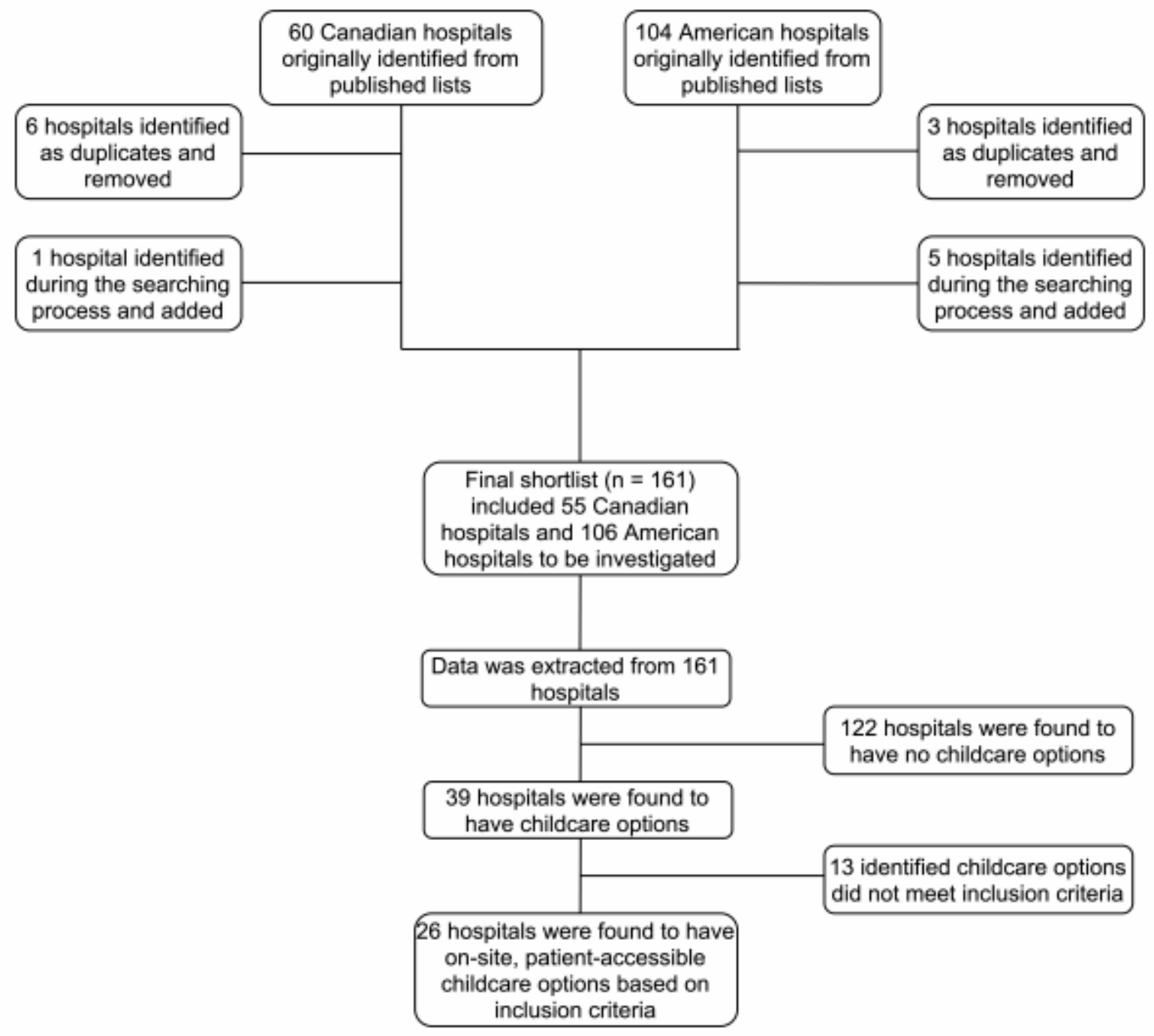

\section{Figure 1}

Flow scheme of the environmental scan 
12

10

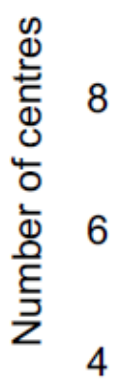

2

0
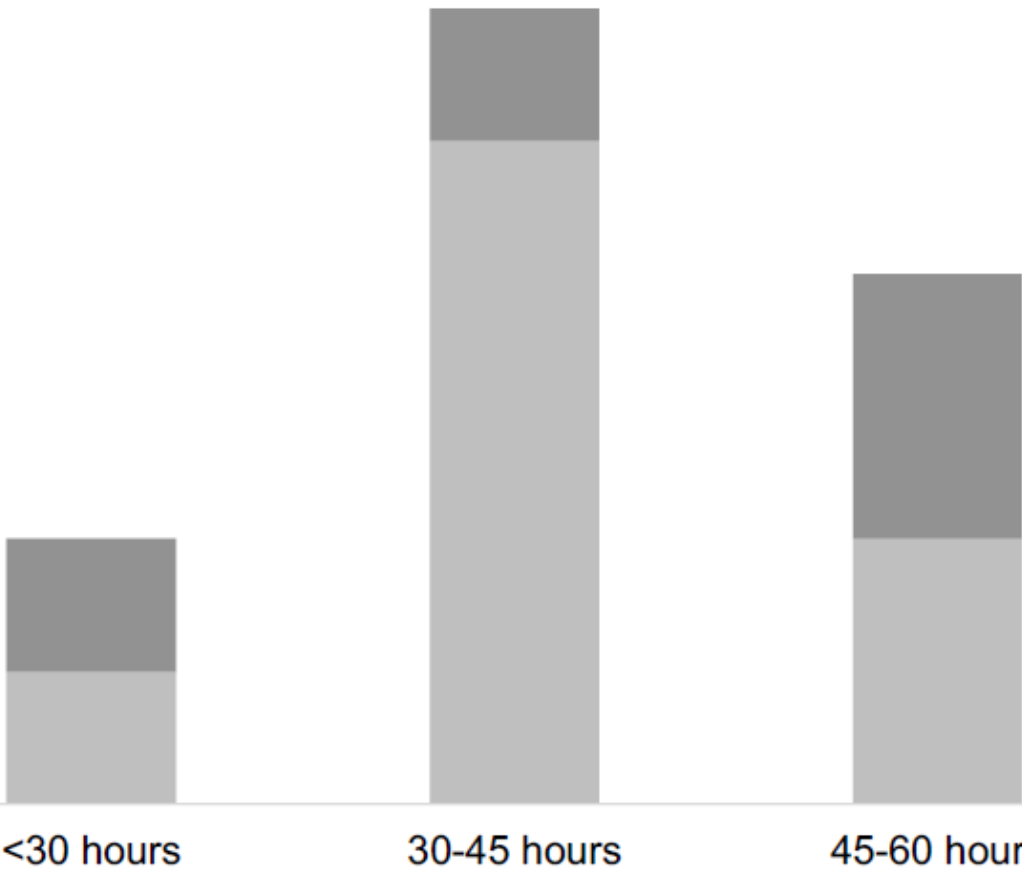

No weekend hours

Weekend hours

30-45 hours

45-60 hours

Variable Hours

Total hours of operation

Figure 2

Total hours of operation of the 26 centres identified, including weekend availability 


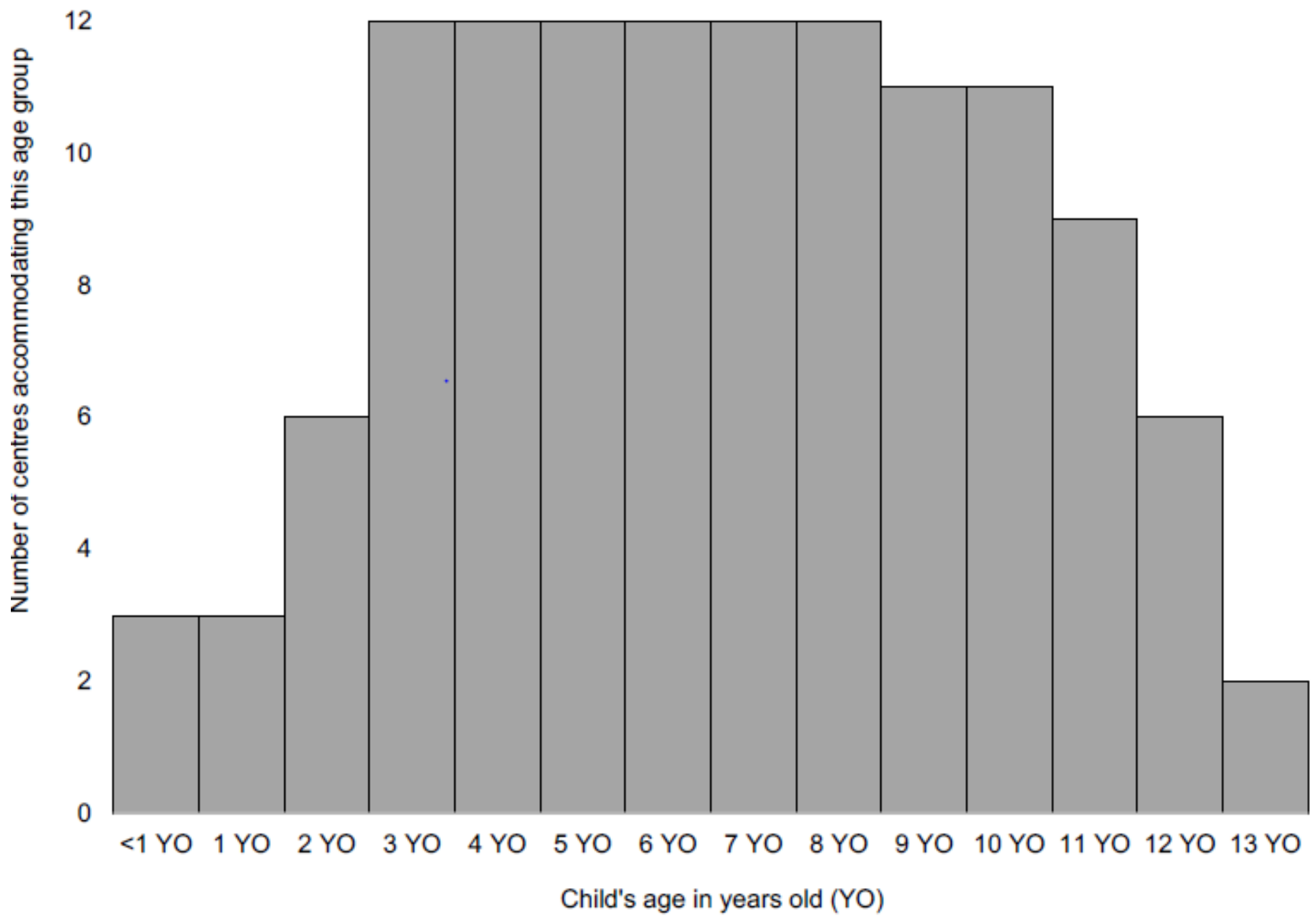

Figure 3

Ages of children accommodated at the centres with fully specified age limits $(n=12)$

\section{Supplementary Files}

This is a list of supplementary files associated with this preprint. Click to download.

- SupplementaryMaterial1.pdf

- SupplementaryMaterial2.xlsx 